\title{
A Comparative Study of High-Dose Colistin Administration for the Management of Multidrug-Resistant Gram-Negative Infections in the ICU
}

\author{
Monireh Ghazaeian, ${ }^{1}$ Majid Mokhtari, ${ }^{2}$ Mehran Kouchek, ${ }^{2}$ Mir-Mohammad Miri, ${ }^{2}$ \\ Reza Goharani, ${ }^{2}$ and Mohammad Sistanizad $\mathbb{D}^{3,4}$ \\ ${ }^{1}$ Department of Clinical Pharmacy, Faculty of Pharmacy, Mazandaran University of Medical Sciences, Sari, Mazandaran, Iran \\ ${ }^{2}$ Department of Pulmonary and Critical Care Medicine, Imam Hossein Teaching and Educational Center, \\ Shahid Beheshti University of Medical Sciences, Tehran, Iran \\ ${ }^{3}$ Department of Clinical Pharmacy, Faculty of Pharmacy, Shahid Beheshti University of Medical Sciences, Tehran, Iran \\ ${ }^{4}$ Prevention of Cardiovascular Disease Research Center, Shahid Beheshti University of Medical Sciences, Tehran, Iran
}

Correspondence should be addressed to Mohammad Sistanizad; sistanizadm@sbmu.ac.ir

Received 1 May 2020; Revised 12 July 2020; Accepted 31 July 2020; Published 21 August 2020

Academic Editor: Peter J. Weina

Copyright $\odot 2020$ Monireh Ghazaeian et al. This is an open access article distributed under the Creative Commons Attribution License, which permits unrestricted use, distribution, and reproduction in any medium, provided the original work is properly cited.

\begin{abstract}
The aim of this study was to assess the clinical and microbiological efficacy and toxicity of different high-dose regimens of colistin in the ICU patients with colistin-sensitive MDR Gram-negative infections. In this prospective, open label, randomized clinical trial, patients with clinical features of infection and positive culture for MDR colistin-sensitive Gram-negative bacteria were randomly allocated to receive colistin, 3 or 9 million units every 8 and 24 hours, (groups A and B), respectively. For each dose regimen, clinical and microbiological response, the rates of nephrotoxicity, and its risk factors were analyzed. Forty-three patients were enrolled, and 35 completed the study protocol, of whom 30 (88.2\%) had ventilator-associated pneumonia (VAP) and 5 (14\%) had bacteremia. Although there were no statistically significant differences in the clinical or microbiological response between the study groups, the microbiological response favored the divided dose group numerically $(p=0.40)$. Clinical response was achieved in 27 of $35(77.1 \%)$ patients $(p=0.999)$. Twelve (34.28\%) patients developed AKI during colistin treatment, 4 and 8 in groups A and B, respectively ( $p$ value $=0.193$ ). Significant risk factors for nephrotoxicity related to colistin were age, hyperbilirubinemia, and coadministration of other nephrotoxic agents. In multivariate regression analysis, the only independent risk factor for CMSassociated AKI was hyperbilirubinemia ( $p$ value $=0.008$ ). Although the clinical and microbiological responses to colistin administration were not statistically different in two groups, the microbiological response favored the divided dose regimen group numerically. We did not observe any significant safety concerns with high-dose colistin administration in this population.
\end{abstract}

\section{Introduction}

The increasing global prevalence of microbial resistance to antibiotics is one of the main challenges facing modern medicine. The Infectious Diseases Society of America (IDSA) recognizes antimicrobial resistance as "one of the greatest threats to human health worldwide."

Multidrug-resistant (MDR) infections, defined as infections caused by the microbial pathogens resistant to two or more classes of antibiotics, has significant adverse effects on morbidity, mortality, and health economy which extend into all aspects of medicine [1]. The emergence of carbapenem-resistant Gram-negative bacteria is of particular concern, as there are limited therapeutic options available for effective treatment of these microorganisms in critically ill patients.

This has led to the redeployment of the older and more toxic antibiotics, such as colistin (polymyxin E), which was discovered nearly 60 years ago. This antimicrobial agent is currently the mainstay of treatment for the resistant Gram- 
negative infections. However, it was not in common use until the 1980s, due to its nephrotoxicity, except in the management of severe Gram-negative infections in patients with cystic fibrosis [2, 3].

Colistin exhibits concentration-dependent bactericidal activity against Gram-negative organisms such as Pseudomonas aeruginosa, Kelbsiella pneumonii, and Acinetobacter baumannii [4-6]. Some evidence showed that the AUC/MIC ratio was the best pharmacokinetic index of cloistin therapeutic effects on Pseudomonas aeruginosa infection [7, 8]. However, significant pharmacokinetic parameters such as $\mathrm{C} \max / \mathrm{MIC}$ ratio, AUC/MIC, and $\mathrm{T}>$ MIC, which define antibiotic efficacy and safety, have not been clearly elucidated for colistin in critically ill patients $[6,9,10]$. Regarding dosing regimens, administration at different intervals of 8,12 , and 24 hours did not show any difference in bacterial killing except lower rate of bacterial regrowth with an 8 hourly regimen [8]. In another study of a critically ill setting, 2 million international unit administration at 8 hours of interval caused inadequate plasma and BAL concentration [9].

The scarcity of data on polymyxin dosing and administrating intervals in the critically ill patient may result in suboptimal dosing, with deleterious effects on patients' outcome and antimicrobial stewardship strategies [3, 9-11].

In order to address these issues, prior studies have recommended to increase the daily doses of colistin from 3 million international units (MIU) in 24 hours to 9 MIU daily, administered in three different dosing schedules of 3 MIU every 8 hours, 4.5 MIU every 12 hours, or 9 MIU daily [12-14].

The primary objective of our study was to evaluate the safety and efficacy of high-dose intravenous (IV) colistin (9 MIU/day in two different dosing intervals), for the treatment of critically ill patients infected with MDR Gramnegative pathogens sensitive to colistin. In addition, we sought to determine the risk factors associated with colistininduced nephrotoxicity in this patient population.

\section{Patients and Methods}

This prospective, randomized clinical trial (RCT) was conducted between May 2014 and Aug 2015 in a general ICU of a 550-bed university hospital in Tehran, Iran. The study was approved by the ethics committee of Shahid Beheshti University of Medical Science and registered at the Iranian Registry of Clinical Trials (IRCT 2014062510178N7).

2.1. Patients. All critically ill ICU patients, 18 years and above, with nosocomial infections, including ventilator-associated pneumonia (VAP) or bacteremia caused by MDR Gram-negative bacteria, were included in the study. VAP was defined based on the criteria set by the American Thoracic Society Consensus Conference on VAP [15]. The Clinical Pulmonary Infection Score (CPIS) was applied to improve the specificity of the clinical diagnosis of pneumonia. The CPIS combines clinical, radiographic, physiologic, and microbiologic data into a numerical result [16]. Sepsis was defined according to the 2013 SCCM/ESICM/
ACCP/ATS/SIS international sepsis definitions conference [17].

The exclusion criteria were pregnancy, breastfeeding, a body mass index of $\geq 35 \mathrm{~kg} / \mathrm{m}^{2}$, creatinine clearance $<40 \mathrm{ml} /$ min (calculated by the Cockcroft-Gault (CG) formula), an alanine aminotransferase or aspartate aminotransferase level $>8$-fold the upper limit of normal, bilirubin $>4 \mathrm{mg} / \mathrm{Dl}$, colistin treatment for $<3$ days after enrollment, and history of colistin administration during the current admission prior to randomization.

Colistin (9 MIU/day) was administered as Colistimethate Sodium (CMS; Colomycin ${ }^{\circledR}$; Forest Laboratories, United Kingdom) in two different dosing intervals of 9 MIU every $24 \mathrm{~h}$ (group A) and 3 MIU every $8 \mathrm{~h}$ (group B). Each dose was prepared in a $100 \mathrm{~mL}$ of sterile isotonic saline solution and was infused over 30 minutes in all study patients.

We recorded the following patient characteristics: age, sex, vital signs, the Acute Physiology and Chronic Health Evaluation (APACHEII) score on admission, the type and site of infection, serum PCT levels, susceptibility to CMS based on the minimum inhibitory concentration (MIC), daily doses and duration of CMS therapy, the cumulative dose of CMS, coadministered antibiotics, administration of other nephrotoxic agents, need for vasoactive and inotropic agents, discontinuation of CMS due to Acute Kidney Injury (AKI), clinical and microbiological responses, and the ICU or hospital 28-day outcome.

The Cockcroft-Gault equation was used to estimate the creatinine clearance to follow the CMS nephrotoxicity. Neurotoxicity was evaluated by the clinical assessment of neurological status whenever possible.

Follow-up samples from the tracheal aspirates and blood were obtained on the fourth and seventh days of the study and when clinically indicated until discharge from the ICU or death. Identification of all causative microorganisms was made using the routine microbiological methods. Susceptibility to CMS was determined by a microdilution technique using the Clinical and Laboratory Standards Institute (CLSI) criteria. Isolates were considered to be susceptible to CMS, if the $\mathrm{MIC}$ was $\leq 2 \mu \mathrm{g} / \mathrm{ml}[18]$.

Clinical response was considered to have been achieved if the signs, symptoms, radiographic findings, and laboratory parameters of infection had been resolved. Bacteriological response was defined as the clearance of Gram-negative bacteria on cultures during or at the completion of the treatment period.

The primary outcome measures were the clinical response and ICU or 28-day mortality. The secondary outcomes were microbiological response and adverse CMS reactions.

Serum creatinine levels and the estimated creatinine clearance were recorded at the baseline and until the end of CMS therapy or death. The baseline glomerular filtration rate (GFR) was calculated by MDRD-4 [19]. The RIFLE criteria were used to stratify AKI into categories of Risk, Injury, Failure, Loss, and End-Stage Renal Disease [20]. AKI during CMS treatment corresponded to the injury stage of the RIFLE criteria. These criteria had to be fulfilled for at 


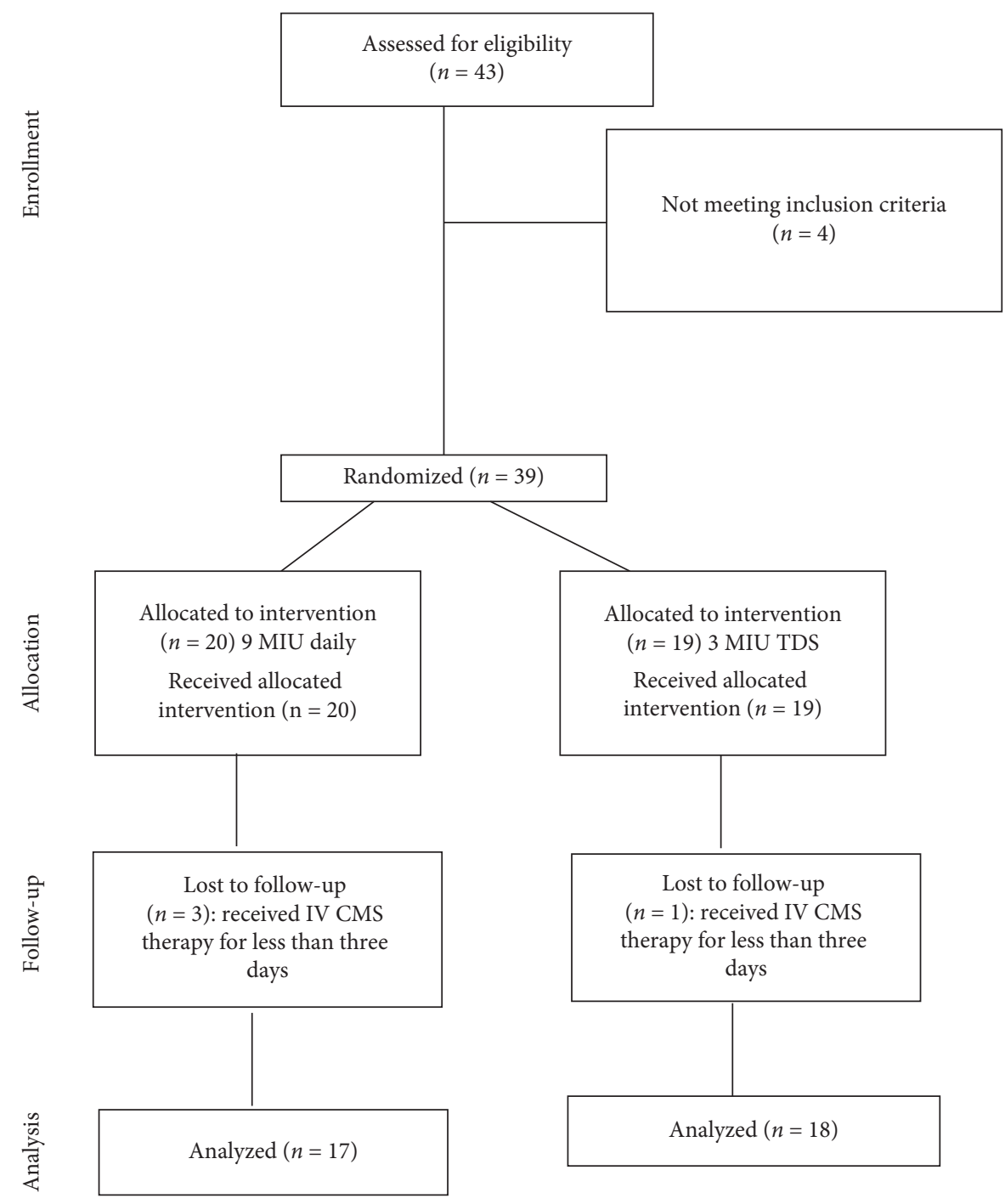

FIgURE 1: Study diagram. CMS, colistimethate sodium.

least 2 continuative determinations, each 24 hours apart and after 48 hours or more of CMS therapy. Patients who developed AKI were followed up closely after the end of CMS therapy to assess the reversibility of their renal function.

The Cockcroft and Gault creatinine clearance estimation was applied to adjust CMS doses during the study period. For a creatinine clearance of 20 to $40 \mathrm{~mL} / \mathrm{min}$, a maintenance dose of CMS $6 \mathrm{MIU} /$ day (group A) or $3 \mathrm{MIU}$ every $12 \mathrm{~h}$ (group B) was administered, and for a creatinine clearance of $<20 \mathrm{~mL} / \mathrm{min}, 3 \mathrm{MIU}$ every $24 \mathrm{~h}$ (groups A and B) was administered. For patients who required renal replacement therapy, a postdialysis maintenance dose of 3 MIU every $48 \mathrm{~h}$ was administered.

2.2. Sample Size. In previous studies, the nephrotoxicity of colistin has been reported as high as 53\% [21]. We proposed that the administration of the total daily dose of colistin as once daily could decrease the nephrotoxicity to $15 \%$. Considering baseline $p=0.53$, comparison $p=0.15$, $\alpha=0.05$, and $\beta=0.8$, the sample size was calculated to be 19 subjects in each group.

2.3. Statistical Analysis. Continuous variables were expressed as mean \pm SD or median (IQR), and categorical variables were expressed as frequencies (percentages). Data were compared between two study groups using the independent sample $t$-test, Mann-Whitney test, and chisquare test or Fisher's exact test for univariate analysis. Adjusted effects of factors on AKI were determined by multiple logistic regression, and significant factors in the univariate analysis were included in multiple logistic regression. The association between serum creatinine changes and CMS therapy duration was assessed by the Pearson correlation coefficient. $p$ values less than 0.05 were 
considered statistically significant. All analyses were performed using SPSS statistical software version 21 (IBM, Armonk, NY, USA).

\section{Results}

CMS was administered to 43 critically ill patients with colistin-sensitive Gram-negative MDR infections. Thirty-five patients (66\% male), completed the study protocol (Figure 1). The baseline characteristics of the patients were not statistically different between study groups (Table 1).

3.1. Bacterial Isolates and Sensitivity Patterns. The isolated Gram-negative pathogens were Acinetobacter baumannii in 30 (86\%) patients, Pseudomonas aeruginosa in 4 (12\%) patients, and Klebsiella pneumonia carbapenemase (KPC) in 1 (2\%) patient. Thirty patients (86\%) received CMS for VAP, and the remaining $5(14 \%)$ received it for bloodstream infections (BSIs). Twenty-one (60\%) bacterial isolates were sensitive to colistin only, and the remaining 14 (40\%) showed either full or intermediate susceptibility to gentamicin, amikacin, or levofloxacin.

In group A, 4 bacterial isolates were also sensitive to gentamicin, 4 were intermediately susceptible to levofloxacin and ciprofloxacin, and 1 was intermediately susceptible to gentamicin. The remaining 7 isolates were only sensitive to colistin. In group B, 4 bacterial isolates were sensitive to gentamicin or amikacin and 1 was intermediately susceptible to ciprofloxacin. The remaining 13 isolates were sensitive only to colistin. The median duration of CMS treatment was 14 days (IQR, 4) for group A and 14 days (IQR, 10) for group B. Carbapenems, aminoglycosides, betalactams, and fluoroquinolones were used in synergy with CMS, for a maximum of 5 days, in $43 \%, 26 \%, 14 \%$, and $17 \%$ of patients, respectively.

3.2. CMS Efficacy and Safety. The clinical response was achieved in 13 (76.5\%) of the 17 patients in group A and 14 $(77.8 \%)$ of the 18 patients in group B. Superinfection by an inherently colistin-resistant organism (Proteus mirabilis) was detected in 2 patients on days 5 and 10 of CMS treatment, one in each study group.

The differences in serum PCT levels were not statistically significant between the two groups $(p=0.468)$.

Nephrotoxicity was observed in 4 patients ( 2 in the first and 2 in the second week) in group A and 8 patients ( 5 in the first and 3 in the second week) in group B. Nephrotoxicity resolved completely in all patients at the end of their ICU stay with CMS dose adjustment, without any patient requiring renal replacement therapy.

The ICU and 28-day mortality rates were $28.6 \%$ and $11.4 \%$, respectively.

In the subgroup of patients who developed AKI, the median onset time in group A was 7 days, and in group B, it was 4 days. The serum creatinine levels before the initiation and at the end of CMS treatment, clinical and microbiological responses, and CMS toxicity were compared between the two study groups (Table 2).
Risk factors for the development of nephrotoxicity during CMS therapy were also evaluated (Table 3).

We were unable to evaluate the evidence of neurotoxicity such as paresthesia, neuromuscular blockade, and apnea as our patients were on mechanical ventilation and ICU sedation. We did not observe any hypersensitivity reaction during the CMS treatment in our study patients.

\section{Discussion}

Due to the limited availability of polymyxin B and the scarcity of clinical data on this agent, CMS (polymyxin E) is currently the main form of polymyxin used worldwide for the management of resistant Gram-negative infection in critically ill ICU patients. CMS is a prodrug with significant toxicity, primarily due to the large doses required to achieve adequate active drug levels, after its conversion in the body.

The optimal dosing regimen of CMS in critically ill patients has not been well established, mainly due to the lack of concrete data on its pharmacokinetics (PK) and pharmacodynamic (PD) properties. Some PK studies have provided valuable data about CMS, supporting the use of larger doses given at longer intervals with higher serum concentrations in critically ill patients [2, 13, 22]. However, other studies have found extended interval regimens of CMS to increase the prevalence of resistant microorganisms, perhaps due to the negligible or absent postantibiotic effect (PAE) of this agent [8].

In our study, the intravenous administration of CMS in 3 MIU every 8 hours or 9 MIU in once-daily doses did not lead to any statistically significant differences in the clinical response. However, the microbiological response, based on microorganism clearance in the blood or sputum, favored the divided dose regimen over the once-daily dose numerically, with values of $61 \%$ vs. $47 \%$, respectively $(p=0.999)$.

The mean APACHE II scores and the number of patients who died within 28 days of ICU admission were 16.33 $( \pm 3.51)$ and 3 in the CMS 9 MIU/day group and 22 and 1 in the CMS $3 \mathrm{MIU}$ three times/day group. Although the mortality rates and the microbiological clearance, despite the higher APACHE II scores, were numerically in favor of the patients who received the divided dose regimen, these values did not reach statistical significance. This could be explained by the small sample size of our study.

The MIC trends were followed up in two patients in each group. Of these patients, two belonging to the CMS $9 \mathrm{MIU}$ daily group expired, with the MIC values showing rising trends above 2 after day 14 of the CMS administration. The MIC levels in the other 2 patients (CMS 3 MIU three times/ day group) remained consistently below 2 . One survived his ICU stay, and the other expired due to other coinfections with no evident rise in the MIC levels.

Higher doses of CMS are associated with safety concerns, especially nephrotoxicity. Various studies have reported different rates of nephrotoxicity ranging from $0 \%$ to $55 \%$ $[4,23-25]$. In the present study, the rate of CMS nephrotoxicity, based on our definition, was $34.3 \%$. In several studies [26-28], numerous factors have been reported to 
TABle 1: Patients' characteristics data.

\begin{tabular}{|c|c|c|c|}
\hline & $\begin{array}{l}\text { Colistin dosing } \\
9 \text { MIU daily }\end{array}$ & 3 MIU TDS & $p$ value \\
\hline \multicolumn{4}{|l|}{ Gender } \\
\hline Male, $n(\%)$ & $11(64.7 \%)$ & $12(66.7 \%)$ & 0.999 \\
\hline Female, $n(\%)$ & $6(35.3 \%)$ & $6(33.3 \%)$ & \\
\hline Age (mean, range) & $45.6 \pm 18.29(20-75)$ & $54.6 \pm 14.61(26-78)$ & 0.115 \\
\hline \multicolumn{4}{|l|}{ Comorbidity } \\
\hline Diabetes mellitus & $2(11.8 \%)$ & $5(27.8 \%)$ & 0.402 \\
\hline $\mathrm{HTN}$ & $5(29.4 \%)$ & $6(33.3 \%)$ & 0.999 \\
\hline Cerebral infarct or hemorrhage & $2(11.8 \%)$ & $4(22.2 \%)$ & $0 . .658$ \\
\hline Other CNS complications & $1(5.9 \%)$ & $3(16.7 \%)$ & 0.603 \\
\hline Heart dysfunction & $0(0 \%)$ & $1(5.3 \%)$ & 0.999 \\
\hline Solid organ transplantation & $3(14.7 \%)$ & $4(22.2 \%)$ & 0.999 \\
\hline Chronic pulmonary disease & $1(5.9 \%)$ & $1(5.3 \%)$ & 0.999 \\
\hline \multicolumn{4}{|l|}{ Admission diagnosis } \\
\hline Cerebral infarct or hemorrhage & $6(35.3 \%)$ & $8(44.4 \%)$ & \\
\hline Other CNS complications & $6(35.3 \%)$ & $5(27.8 \%)$ & \\
\hline Trauma & $1(5.9 \%)$ & $1(5.6 \%)$ & 0.986 \\
\hline GI bleeding & $1(5.9 \%)$ & $1(5.6 \%)$ & \\
\hline HTN crisis & $3(17.6 \%)$ & $3(16.7 \%)$ & \\
\hline APACHE II score, mean \pm SD & $21 \pm 4.96$ & $20.4 \pm 4.99$ & 0.723 \\
\hline CMS duration, median days (IQR) & $14(4)$ & $14(10)$ & 0.369 \\
\hline ICU duration, median days (IQR) & $31(17)$ & $29.5(30)$ & 0.859 \\
\hline ICU mortality, all days, $n(\%)$ & $6(35.29 \%)$ & $4(22.22 \%)$ & 0.392 \\
\hline Source of infection & & & 0.581 \\
\hline BSI, $n(\%)$ of cases & $3(17.6 \%)$ & $2(11.1 \%)$ & \\
\hline $\mathrm{VAP}, n(\%)$ of cases & $14(82.4 \%)$ & $16(88.9 \%)$ & \\
\hline \multicolumn{4}{|l|}{ Combination with other antibiotics } \\
\hline Aminoglycosides & 3 & 6 & \\
\hline Beta-lactams & 3 & 2 & \\
\hline Quinolones & 3 & 3 & \\
\hline Carbapenems & 8 & 7 & \\
\hline
\end{tabular}

APACHE, Acute Physiology and Chronic Health Evaluation; BSI, bloodstream infection; CMS, colistimethate sodium; ICU, intensive care unit; MIU, million international units; SD, standard deviation; CNS, central nervous system, CVA, cerebral vascular accident; GI, gastrointestinal; HTN, hypertension; VAP, ventilator-associated pneumonia. MIN; minimum, MAX; maximum. IQR; interquartile range.

TABLE 2: Clinical and microbiological response and toxicity comparison.

\begin{tabular}{|c|c|c|c|c|}
\hline & 9 MIU daily, $n(\%)$ & $3 \mathrm{MIU}$ TDS, $n(\%)$ & Total, $n(\%)$ & $p$ value \\
\hline Clinical response & $13(76.5 \%)$ & $14(77.8 \%)$ & $27(77.1 \%)$ & 0.999 \\
\hline Microbiological response & $8(47.1 \%)$ & $11(61.1 \%)$ & $19(54.3 \%)$ & 0.404 \\
\hline AKI & $4(23.52 \%)$ & $8(44.44 \%)$ & $12(34.28 \%)$ & 0.193 \\
\hline Time to increase Scr* & $7(7.5)$ & $4.5(4.5)$ & & 0.283 \\
\hline Time to response* & $14(3)$ & $14(10)$ & $14(6)$ & 0.720 \\
\hline
\end{tabular}

*Median day (IQR), AKI; acute kidney injury, IQR; interquartile range.

increase the risk of CMS-associated nephrotoxicity. In bivariate analysis of the patients who developed AKI, significant risk factors were age, hyperbilirubinemia, and coadministration of other nephrotoxic agents. However, in multivariate regression analysis, the only independent risk factor for CMS-associated AKI was hyperbilirubinemia ( $p$ value $=0.008)$ (Table 3$)$. This association could have been due to hyperbilirubinemia causing false elevation of creatinine without significant impact on the actual GFR.

There are some data supporting the use of high-dose CMS, with extended intervals, for reaching the maximum efficacy with lower rates of toxicity due to its concentration- dependent properties $[14,24]$. Although we found no statistically significant differences between the two dosing regimens regarding the rates of nephrotoxicity, the serum creatinine elevation above the baseline occurred earlier in the divided dose regimen (Table 2).

The differences in our study groups regarding serum creatinine changes from the baseline to the peak did not reach the statistical significance (Figure 2).

The limitations of our study were the small sample size, unblinded design, lack of therapeutic drug monitoring of CMS and PK parameters determination, the absence of serial MIC data in all patients, and short duration of follow-up. 
TABLE 3: Risk factors for the development of nephrotoxicity during CMS therapy.

\begin{tabular}{|c|c|c|c|c|}
\hline Risk factors & NO AKI $(N=23)$ & AKI $(N=12)$ & $p$ value & $p$ value-adjusted \\
\hline Age $($ mean $\pm S D)$ & $45.9 \pm 16.71$ & $58.5 \pm 14.48$ & 0.034 & 0.753 \\
\hline Male, $n(\%)$ & $14(60.9 \%)$ & $9(75 \%)$ & 0.403 & \\
\hline APACHE II score $($ mean \pm SD) & $20.4 \pm 5.26$ & $21.2 \pm 4.59$ & 0.687 & \\
\hline ICU duration, median days (IQR) & $28(21)$ & $36.5(22)$ & 0.190 & \\
\hline CMS duration, median days (IQR) & $14(8)$ & $14(19)$ & 0.604 & \\
\hline Number of nephrotoxic agents ${ }^{1}$ & & & & 0.178 \\
\hline 0 & $13(56.5 \%)$ & $2(16.7 \%)$ & & \\
\hline 1 & $5(21.7 \%)$ & $5(41.7 \%)$ & & \\
\hline 2 & $5(21.7 \%)$ & $2(16.7 \%)$ & 0.028 & \\
\hline 3 & $0(0 \%)$ & $2(16.7 \%)$ & & \\
\hline 4 & $0(0 \%)$ & $1(8.3 \%)$ & & \\
\hline $\mathrm{DM}, n(\%)$ & $3(13 \%)$ & $4(33.3 \%)$ & 0.200 & \\
\hline HTN, $n(\%)$ & $5(21.7 \%)$ & $6(50 \%)$ & 0.087 & \\
\hline COPD, $n(\%)$ & $1(4.3 \%)$ & $3(25 \%)$ & 0.106 & \\
\hline Hypoalbuminaemia $^{2}$, mean serum level $\pm S D$ & $3.0 \pm 0.45$ & $2.8 \pm 0.57$ & 0.235 & \\
\hline AST, mean serum level \pm SD & $68 \pm 53.9$ & $93.9 \pm 83.7$ & 0.536 & \\
\hline $\mathrm{ALT}$, mean serum level $\pm \mathrm{SD}$ & $73.3 \pm 59.8$ & $93.9 \pm 83.7$ & 0.418 & \\
\hline Hyperbilirubinaemia $^{3}$, mean serum level \pm SD & $1.2 \pm 0.88$ & $3.0 \pm 2.49$ & 0.029 & 0.008 \\
\hline
\end{tabular}

APACHE, Acute Physiology and Chronic Health Evaluation; CMS, colistimethate sodium; ICU, intensive care unit; SD, standard deviation; DM, diabetes mellitus; HTN, hypertension; COPD, chronic obstructive pulmonary disease; AST, alanine aminotransferase; ALT, aspartate aminotransferase, IQR; interquartile range. ${ }^{1}$ Nephrotoxic agents include vancomycin, aminoglycosides, angiotensin-converting enzyme inhibitors, diuretics, radio contrast agents, and vasopressors. ${ }^{2}$ Defined as a serum albumin level $<3 \mathrm{~g} / \mathrm{dL}$. ${ }^{3}$ Defined as a total bilirubin level $>5 \mathrm{mg} / \mathrm{dL}$.

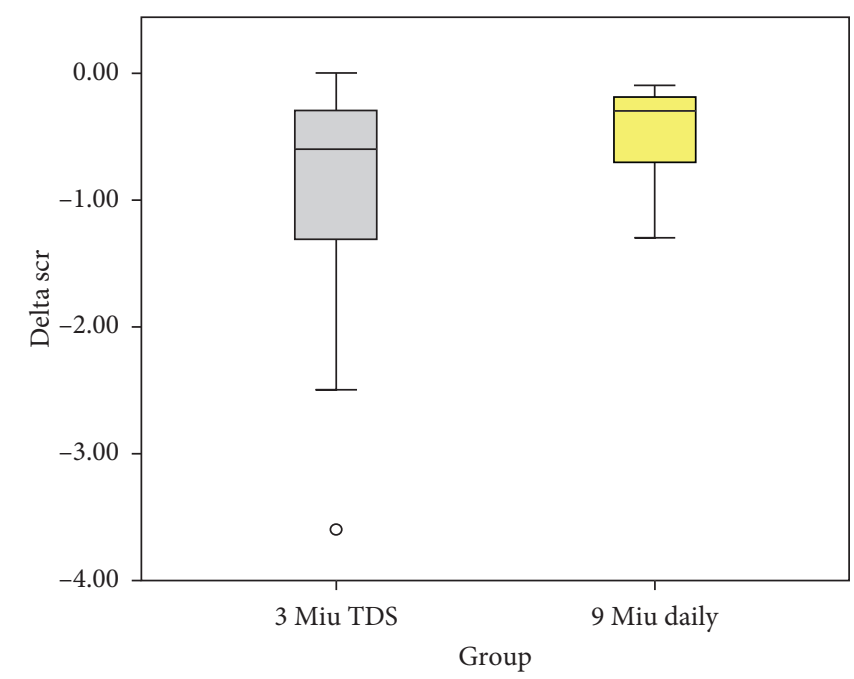

Figure 2: Box plot of SCr changes from baseline levels to the peak.

\section{Conclusions}

The administration of high-dose CMS, i.e., 9 MIU/day or 3 MIU three times/day, for the management of MDR Gramnegative pathogens in critically ill patients can be a reasonable option with acceptable efficacy and safety profile.

The risk factors for development of nephrotoxicity with CMS, such as age, serum bilirubin levels, and coadministration of other nephrotoxic agents, should be considered in this patient population.

Further investigations with a larger sample size, therapeutic drug monitoring of CMS, more astringent microbiological assessments, and longer patient follow-up are warranted for expanding our knowledge of the efficacy and safety of CMS in higher doses for the management of MDR Gram-negative pathogens in the critically ill population.

\section{Abbreviations}

MDRD: Modification of diet in renal disease

AKI: Acute kidney injury.

\section{Data Availability}

The data that support the findings of this study are available from the corresponding author upon reasonable request. 


\section{Conflicts of Interest}

There are no conflicts of interest to declare.

\section{Acknowledgments}

This study was funded by a grant from vice chancellery for research affairs of Shahid Beheshti University of Medical Sciences (grant no. SBMU-1394-2021).

\section{References}

[1] D. V. Duin, K. S. Kaye, E. A. Neuner, and R. A. Bonomo, "Carbapenem-resistant enterobacteriaceae: a review of treatment and outcomes," Diagnostic Microbiology and Infectious Disease, vol. 75, no. 2, pp. 115-120, 2013.

[2] R. L. Nation and J. Li, "Colistin in the 21st century," Current Opinion in Infectious Diseases, vol. 22, no. 6, pp. 535-543, 2009.

[3] L. M. Lim, N. Ly, D. Anderson et al., "Resurgence of colistin: a review of resistance, toxicity, pharmacodynamics, and dosing," Pharmacotherapy: The Journal of Human Pharmacology and Drug Therapy, vol. 30, no. 12, pp. 1279-1291, 2010.

[4] P. Koomanachai, S. Tiengrim, P. Kiratisin, and V. Thamlikitkul, "Efficacy and safety of colistin (colistimethate sodium) for therapy of infections caused by multidrug-resistant Pseudomonas aeruginosa and Acinetobacter baumannii in Siriraj Hospital, Bangkok, Thailand," International Journal of Infectious Diseases, vol. 11, no. 5, pp. 402-406, 2007.

[5] J. Li, J. Turnidge, R. Milne, R. L. Nation, and K Coulthard, "In vitro pharmacodynamic properties of colistin and colistin methanesulfonate against Pseudomonas aeruginosa isolates from patients with cystic fibrosis," Antimicrobial Agents and Chemotherapy, vol. 45, no. 3, pp. 781-785, 2001.

[6] J. Li, C. R. Rayner, R. L. Nation et al., "Heteroresistance to colistin in multidrug-resistant Acinetobacter baumannii," Antimicrobial Agents and Chemotherapy, vol. 50, no. 9, pp. 2946-2950, 2006.

[7] R. V. Dudhani, J. D. Turnidge, K. Coulthard et al., "Elucidation of the pharmacokinetic/pharmacodynamic determinant of colistin activity against Pseudomonas aeruginosa in murine thigh and lung infection models," Antimicrobial Agents and Chemotherapy, vol. 54, no. 3, pp. 1117-1124, 2010.

[8] P. J. Bergen, J. Li, R. L. Nation, J. D. Turnidge, K. Coulthard, and R. W. Milne, "Comparison of once-, twice-and thricedaily dosing of colistin on antibacterial effect and emergence of resistance: studies with Pseudomonas aeruginosa in an in vitro pharmacodynamic model," Journal of Antimicrobial Chemotherapy, vol. 61, no. 3, pp. 636-642, 2008.

[9] R. Imberti, M. Cusato, P. Villani et al., "Steady-state pharmacokinetics and BAL concentration of colistin in critically ill patients after IV colistin methanesulfonate administration," Chest, vol. 138, no. 6, pp. 1333-1339, 2010.

[10] A. S. Michalopoulos and M. E. Falagas, "Colistin: recent data on pharmacodynamics properties and clinical efficacy in critically ill patients," Annals of Intensive Care, vol. 1, no. 1, pp. 1-6, 2011.

[11] J. Garnacho-Montero, C. Ortiz-Leyba, F. J. Jimenez-Jimenez et al., "Treatment of multidrug-resistant Acinetobacter baumannii ventilator-associated pneumonia (VAP) with intravenous colistin: a comparison with imipenem-susceptible VAP," Clinical Infectious Diseases, vol. 36, no. 9, pp. 1111-1118, 2003.
[12] G. L. Daikos, A. Skiada, J. Pavleas et al., "Serum bactericidal activity of three different dosing regimens of colistin with implications for optimum clinical use," Journal of Chemotherapy, vol. 22, no. 3, pp. 175-178, 2010.

[13] N. Markou, S. L. Markantonis, E. Dimitrakis et al., "Colistin serum concentrations after intravenous administration in critically ill patients with serious multidrug-resistant, gramnegative bacilli infections: a prospective, open-label, uncontrolled study," Clinical Therapeutics, vol. 30, no. 1, pp. 143-151, 2008.

[14] L. Dalfino, F. Puntillo, A. Mosca et al., "High-dose, extendedinterval colistin administration in critically ill patients: is this the right dosing strategy? A preliminary study," Clinical Infectious Diseases, vol. 54, no. 12, pp. 1720-1726, 2012.

[15] Society AT and America IDSo, "Guidelines for the management of adults with hospital-acquired, ventilator-associated, and healthcare-associated pneumonia," American Journal of Respiratory and Critical Care Medicine, vol. 171, no. 4, pp. 388-416, 2005.

[16] J.-J. Parienti, M. Ramakers, and P. Charbonneau, "Shortcourse empiric antibiotic therapy for patients with pulmonary infiltrates in the intensive care unit," American Journal of Respiratory and Critical Care Medicine, vol. 164, no. 1, pp. 172-173, 2001.

[17] R. P. Dellinger, M. M. Levy, A. Rhodes et al., "Surviving sepsis campaign: international guidelines for management of severe sepsis and septic shock: 2012," Critical Care Medicine, vol. 41, no. 2, pp. 580-637, 2013.

[18] P. Wayne and Clinical and Laboratory Standards Institute, "Implementation guide of poct for health care providers," pp. 1-37, 2007.

[19] G. Eknoyan and N. Levin, "K/DOQI clinical practice guidelines for chronic kidney disease: evaluation, classification, and stratification-foreword," American Journal of Kidney Diseases, vol. 39, no. 2, pp. S14-S266, 2002.

[20] R. Bellomo, C. Ronco, J. A. Kellum, R. L. Mehta, and P. Palevsky, "Acute renal failure-definition, outcome measures, animal models, fluid therapy and information technology needs: the second international consensus conference of the Acute Dialysis Quality Initiative (ADQI) group," Critical Care, vol. 8, no. 4, pp. R204-R212, 2004.

[21] M. E. Falagas and S. K. Kasiakou, "Toxicity of polymyxins: a systematic review of the evidence from old and recent studies," Critical Care, vol. 10, no. 1, p. R27, 2006.

[22] P. J. Bergen, J. Li, C. R. Rayner, and R. L. Nation, "Colistin methanesulfonate is an inactive prodrug of colistin against Pseudomonas aeruginosa," Antimicrobial Agents and Chemotherapy, vol. 50, no. 6, pp. 1953-1958, 2006.

[23] M. E. Falagas, M. Rizos, I. A. Bliziotis, K. Rellos, S. K. Kasiakou, and A. Michalopoulos, "Toxicity after prolonged (more than four weeks) administration of intravenous colistin," BMC Infectious Diseases, vol. 5, no. 1, p. 1, 2005.

[24] L. Sorlí, S. Luque, S. Grau et al., “Trough colistin plasma level is an independent risk factor for nephrotoxicity: a prospective observational cohort study," BMC Infectious Diseases, vol. 13, no. 1, pp. 1-9, 2013.

[25] J. M. Pogue, J. Lee, D. Marchaim et al., "Incidence of and risk factors for colistin-associated nephrotoxicity in a large academic health system," Clinical Infectious Diseases, vol. 53, no. 9, pp. 879-884, 2011.

[26] J. Kim, K.-H. Lee, S. Yoo, and H. Pai, "Clinical characteristics and risk factors of colistin-induced nephrotoxicity," International Journal of Antimicrobial Agents, vol. 34, no. 5, pp. 434-438, 2009. 
[27] J.-A. Kwon, J. E. Lee, W. Huh et al., "Predictors of acute kidney injury associated with intravenous colistin treatment," International Journal of Antimicrobial Agents, vol. 35, no. 5, pp. 473-477, 2010.

[28] M. Rocco, L. Montini, E. Alessandri et al., "Risk factors for acute kidney injury in critically ill patients receiving high intravenous doses of colistin methanesulfonate and/or other nephrotoxic antibiotics: a retrospective cohort study," Critical Care, vol. 17, no. 4, p. R174, 2013. 